\title{
Kindschaftsrechtliche Aspekte des FGG-Reformgesetzes - Verfahrenseile schadet bei häuslicher Gewalt
}

In sorge- und umgangsrechtlichen Verfahren liegt die Verfahrensdauer an deutschen Gerichten derzeit bei etwa sieben Monaten. Das Bundesverfassungsgericht und der Europäische Gerichtshof für Menschenrechte haben gerade in diesen Verfahren den Faktor Zeit als ausschlaggebend für eine nachhaltige Verwirklichung des Kindeswohls angemahnt und den Gesetzgeber zum Handeln aufgefordert.

\section{Zielsetzung des Reformentwurfes}

Der Regierungsentwurf für ein „Gesetz zur Reform der Verfahren in Familiensachen und in den Angelegenheiten der freiwilligen Gerichtsbarkeit" (FGG$\mathrm{RG}$ ) sieht daher unter anderem vor, dass bestimmte Familien- und Kindschaftssachen, nämliche solche, die den Aufenthalt, die Herausgabe des Kindes, das Umgangsrecht oder Verfahren wegen Gefährdung des Kindeswohls betreffen, ,vorrangig durchzuführen sind“ (vgl. $\mathbb{S}$ 50e Abs. 1 FGG-E; 155 Abs. 1 FGG-RG). Des Weiteren wird ein explizites Beschleunigungsgebot statuiert (vgl. $\mathbb{S} 550$ e Abs. 1 FGG-E; 155 Abs. 1 FGG-RG) und der Fokus auf ein Hinwirken des Gerichts zugunsten einer einvernehmlichen Lösung gesetzt (vgl. $\$ 156$ FGG-RG).

Durch eine rasche Terminierung und durch einen möglichst frühzeitigen Einbezug von Beratungsstellen soll die Verfestigung einer destruktiven Dynamik zu Lasten der betroffenen Kinder und eine Entfremdung vom nicht-betreuenden Elternteil verhindert werden.

Der Entwurf zum FGG-Reformgesetz lehnt sich in der Ausgestaltung des kindschaftsrechtlichen Verfahrens stark an die als „Cochemer Modell“1 entwickelte Praxis an: Der gerichtliche Anhörungstermin soll spätestens einen Monat nach Beginn des Verfahrens stattfinden. In diesem Anhörungstermin hört das Gericht die Eltern und das Jugendamt an, das mündlich in der Verhandlung den aktuellen Sachstand berichtet. Ein schriftlicher Bericht ist nicht vorgesehen. (In der Cochemer Praxis und in weiteren Cochem-nahen Modellen in anderen Amtsgerichtsbezirken wird den Prozessbeteiligten eine Woche zugebilligt, um sich auf schriftlich eingereichte Anträge von Prozessbeteiligten vorzubereiten. Der sonst üblichen schriftlichen Erwiderung bedarf es nicht.) Der beschleunigt angesetzte Termin dient somit der nicht oder nur teilweise schrift- lich vorbereiteten Aufklärung des Sachverhalts und soll im kooperativen Zusammenwirken aller an diesem Termin Beteiligten möglichst zu einer einvernehmlichen Lösung im ersten Termin führen. Dabei geht der Entwurf davon aus, dass es dem Jugendamt in der Frist von einem Monat gelingt, mit den Eltern und den betroffenen Kindern Kontakt aufzunehmen und in der Sitzung eine Stellungnahme abzugeben, die trotz der knappen Vorbereitungszeit so fundiert ist, dass es im ersten Termin bereits zu einem verfahrensbeendenden Vergleich bzw. einer Entscheidung kommen kann.

\section{Kritik zugunsten einer am Kindeswohl orientierten Praxis}

Verfahrensbeschleunigung ist kein Selbstzweck. Das Beschleunigungsgebot soll dem Kindeswohl dienen und wird durch dieses zugleich begrenzt. Es muss daher überprüft werden, ob dieser „beschleunigte“ Verfahrensweg und die Stärkung des Elements der Einvernehmlichkeit in jedem Stadium des Verfahrens tatsächlich im Einzelfall eine optimale Umsetzung des Kindeswohls ermöglichen.

Ein früher erster Termin im Sinn des Vorranggebots ist sicher zu begrüßen, gerade wenn es darum geht, frühzeitig die Verfestigung kindeswohlgefährdender Dynamiken zu vermeiden und somit das Zeitfenster für niederschwellige, unterstützende Angebote der Kinder- und Jugendhilfe offen zu halten. Dem Gesetzentwurf liegt aber auch die Vorstellung zugrunde, dass „nur eine sofortige Regelung ... die Gefahr einer für das Kindeswohl abträglichen Unterbrechung von Umgangskontakten zwischen dem Kind und dem nicht betreuenden Elternteil" vermeidet.

Diese Sicht der Dinge blendet aus, dass tragfähige Lösungen gerade im Interesse des Kindes auch Zeit erfordern können. Ein rasches Wiedereinsetzen des Umgangs um jeden Preis dient nicht in jedem Fall dem Kindeswohl. In gewaltbelasteten Familiensystemen werden Strukturen benötigt, die Zeitfenster für das Herstellen von Schutz und Sicherheit und damit für die Stabilisierung der kindlichen und erwachsenen Gewaltopfer, aber auch für Beratungsprozesse eröffnen. Erst im Verbund

1 Rudolph, Jürgen: Du bist mein Kind. Die „Cochemer Praxis" - Wege zu einem menschlichen Familienrecht, Berlin 2007, S. 47 f.; http://www.ak-cochem.de/.

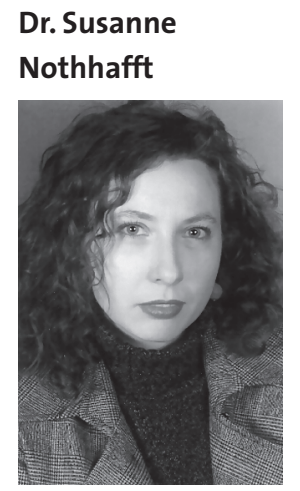

Mltglied der Kommission Gewalt gegen Frauen und Kinder des djb; Wiss. Referentin am Deutschen Jugendinstitut München 
mit Täterprogrammen oder beraterischen Interventionen können dann am Kindeswohl orientierte Konzepte für den Umgang und die elterliche Sorge entwickelt werden.

Im Weiteren gibt es gerade in den kindschaftsrechtlichen Verfahren Fallkonstellationen, die im Hinblick auf ihre Dynamik und Struktur eine andere, „entschleunigte“ Vorgehensweise verlangen. Zu denken ist hierbei an die für Fragen des Umgangsrechts durchaus relevante Abklärung des Vorliegens häuslicher Gewalt oder hochstreitiger Trennungsverläufe sowie den Verdacht auf intrafamiliäre sexualisierte Gewalt.

Der im Rahmen der FGG-Reform für die Kindschaftssachen vorgesehene Verfahrensweg ist daher - insbesondere im Hinblick auf eine optimale Umsetzung des Kindeswohls in grundlegenden Punkten zu kritisieren:

\section{Strukturelle Defizite}

Der Reformentwurf vernachlässigt die offensichtliche Problematik, dass im großstädtischen Bereich - Cochem ist eine Kleinstadt - die institutionellen Strukturen weder finanziell noch personell so ausgestattet sind, dass die knappen Zeitvorgaben von Jugendämtern und Beratungsstellen sinnvoll, also nachhaltig und zum Wohl des Kindes und der von Gewalt betroffenen Frauen eingehalten werden können.

Zudem wurde das Cochemer Modell bislang weder strukturell noch adressatenbezogen evaluiert. Es liegen nur die $\mathrm{Pu}-$ blikationen des Amtsgerichts Cochem vor, die ausweisen, dass zwischen 1998 und 2003 in nahezu 100 Prozent der Fälle zugunsten eines gemeinsamen Sorgerechts entschieden wurde und zwischen 1996 und 1999 keine streitigen Entscheidungen zum Umgangs- und Sorgerecht mehr ergangen sind. ${ }^{2}$ Dies widerspricht zum Beispiel in eklatanter Weise den bundesdeutschen Prävalenzzahlen für das Auftreten von häuslicher Gewalt und für das Misshandlungsrisiko zu Lasten von Frauen und Kindern bei Umgangskontakten nach der Trennung aus einer häuslichen Gewaltbeziehung. ${ }^{3}$

\section{Mehrdimensionale Gefährdungseinschätzung}

Für eine de lege artis mehrdimensionale Gefährdungseinschätzung $^{4}$ sollten nach einer gründlichen Informationssammlung die Aspekte einbezogen werden, welche die individuelle Lebenssituation einer oder eines Minderjährigen und ihrer oder seiner Familie charakterisieren. Erst differenzierende und multiperspektivische Kenntnisse über Persönlichkeit und Lebensgeschichte von Eltern und Kind, über die Besonderheit der Eltern-Kind-Beziehungen sowie der familiären Lebenswelt können die Beantwortung der kind-, eltern- und gefährdungsbezogenen Fragen ermöglichen. Die Relevanz der einzelnen Aspekte ist von der individuellen Fallkonstellation sowie der jeweiligen Einschätzaufgabe abhängig. Beispielsweise kann die Einschätzung einer gravierenden Vernachlässigung eines Kleinkindes von seinen Suchtmittel konsumierenden Eltern aufgrund ausreichender eltern- und kindbezogener Informationen sowie vorhandener ärztlicher Diagnostik möglicherweise recht schnell erfolgen. Der durch eine Tagesstätte gemeldete
Verdacht auf innerfamiliären sexuellen Missbrauch eines siebenjährigen Jungen aufgrund eines sensiblen, das Kind nicht zusätzlich belastenden oder gefährdenden Vorgehens wird hingegen langwieriger und auch schwieriger abzuklären sein. Das Ergebnis einer Gefährdungseinschätzung ergibt sich aus der Zusammenschau, aus der kontextabhängigen Gewichtung und fachlichen Bewertung der einzelnen Dimensionen sowie der Qualität ihrer Wechselwirkungen. Ein solches Vorgehen verlangt ausreichende personelle Ressourcen und Zeit für die Exploration.

\section{Intrafamiliäre sexualisierte Gewalt}

Fallkonstellationen, die einen Verdacht auf intrafamiliäre sexualisierte Gewalt gegen Kinder nahelegen, verlangen zudem ein Vorgehen, das noch weiter von den Vorgaben des Beschleunigungsgebotes entfernt liegen muss.

Eine besondere Schwierigkeit ergibt sich daraus, dass bei einem Anfangsverdacht auf sexualisierte Gewalt häufig nicht ausgeschlossen werden kann, dass Vater, Mutter oder beide selbst Täter oder Täterin sind. In diesem Fall ist es mehr als bei anderen Formen von Kindeswohlgefährdung fraglich, inwieweit der gewalttätige Elternteil bereit ist, Verantwortung für die Taten zu übernehmen und zum Wohle des Kindes zu kooperieren ${ }^{5}$. Im Gegenteil kann - auch im Hinblick auf mögliche strafrechtliche Konsequenzen - ein Interesse bestehen, die Aufklärung zu verhindern. Es kann deshalb vielfach auch nicht ausgeschlossen werden, dass ein offenes Thematisieren des Verdachts auf intrafamiliäre sexualisierte Gewalt gegenüber dem gewalttätigen Elternteil mehr schadet als nützt, weil es den Täter oder die Täterin veranlasst, durch Druck auf das betroffene Kind die Aufdeckung zu erschweren und Hilfe zu verhindern ${ }^{6}$.

Diese besondere Problematik wird im SGB VIII seit dem 1. Oktober 2005 explizit berücksichtigt. Das Gesetz baut zwar nach wie vor stark auf eine Zusammenarbeit mit den Eltern, um diese in ihrer Erziehungskompetenz zu unterstützen und sie zu befähigen, zum Wohl des Kindes zu handeln. Hiervon sind jedoch nunmehr ausdrücklich Ausnahmen vorgesehen,

2 http://www.ak-cochem.de/.

3 BMFSFJ: Sorge- und Umgangsrecht bei häuslicher Gewalt: aktuelle rechtliche Entwicklungen, wissenschaftliche Erkenntnisse und Empfehlungen, Berlin 2002.

4 Lillig, Susanna: „Welche Aspekte können insgesamt bei der Einschätzung von Gefährdungsfällen bedeutsam sein?" In: Kindler, H., Lillig, S., Blüml, H., Meysen, T. \& Werner, A. (Hg.) „Handbuch Kindeswohlgefährdung nach $\S 1666$ BGB und Allgemeiner Sozialer Dienst (ASD)“, München 2006 Deutsches Jugendinstitut e.V., Nr. 73.

5 Verleugnungsstrategien und Abwehrmechanismen von Missbrauchstätern wurden vielfach beschrieben. Der Begriff des „Graugestehens“ z. B. beschreibt ein vordergründiges Zugeben von Taten bei gleichzeitiger Bagatellisierung. Schuld und Verantwortung werden anderen zugeschrieben, negative Folgen des Missbrauchshandelns für betroffene Kinder werden abgestritten, umgedeutet oder minimiert. Vgl.: Deegener, Günther: Sexueller Missbrauch. Die Täter, Weinheim 1995, S. 59 ff. Deegener analysiert in seinen Arbeiten unter dem Titel „Verantwortungs-Abwehr-System“ die ausgeprägte Abwehr der Verantwortungsübernahme von Missbrauchstätern.

6 Zum Geheimhaltungsgebot der Täter vgl. Deegener, Günther: Sexueller Missbrauch an Kindern. Ausmaß, Hindergründe, Folgen, Weinheim 1998, S. $141 \mathrm{ff}$. 
wenn durch das Thematisieren mit den Eltern der wirksame Schutz des Kindes oder der oder des Jugendlichen infrage gestellt bzw. der Zugang zur Hilfe ernsthaft gefährdet würde ( $\int 8$ a Abs. 1 Satz 2, $\mathbb{6} 62$ Abs. 3 Nr. 4 SGB VIII). Dieser Schutzmodus für (möglicherweise) von sexualisierter Gewalt betroffene Kinder und Jugendliche wird durch die Strukturen eines beschleunigten familiengerichtlichen Verfahrens ausgehebelt und konterkariert.

\section{Häusliche Gewalt}

Auch bei Fällen von Männergewalt gegen Frauen und Kinder in Form von häuslicher Gewalt wird die Vorgehensweise, die das FGG-RG vorschlägt, dem Kindeswohl nicht gerecht. In einer Studie des kriminologischen Forschungsinstituts Niedersachsen ${ }^{7}$ gaben 21,3 Prozent der 16- bis 19-Jährigen an, mit elterlicher Partnergewalt konfrontiert zu sein. Die Studien von Hester $^{8}$ zeigen, dass 90 Prozent der Kinder sich bei den gewalttätigen Übergriffen im selben oder im angrenzenden Raum befanden, 73 Prozent beobachteten die Gewalthandlungen zudem direkt. Kinder, welche die Gewalt des Vaters gegen die Mutter miterleben, zeigen die gleichen Störungen in der emotionalen, kognitiven und sozialen Entwicklung wie Kinder, die direkt vom Vater misshandelt werden. ${ }^{9}$ Kinder sind deshalb nie nur Zeugen häuslicher Gewalt, sondern immer auch Opfer. Um einen Umgang zwischen den Kindern und den Tätern häuslicher Gewalt wieder zu ermöglichen, braucht es daher ein sensibles, schrittweises Vorgehen, das den Kindern Zeit und Raum für alters- und geschlechtsspezifische Unterstützungsangebote eröffnet, in denen sie über das Erlebte sprechen und die Gewalterfahrungen bearbeiten können. Zudem müssen die Täter in die Verantwortung genommen und ihnen in spezifischen Täterprogrammen eine Verhaltensänderung ermöglicht werden.

Intrafamiliäre Gewaltbeziehungen sind durch eine spezifische Dynamik von Macht und Ohnmacht gekennzeichnet, die eine kooperative, am Konsens orientierte Einigung autonomer Partner - am selben Tisch und in kurzer Zeit - unmöglich macht. Frauen und Kinder, die von Gewalt betroffen sind, müssen die Chance erhalten, Schutz und Sicherheit zu finden. Eine ungestörte Neuorganisation der Lebenssituation gerade auch im Sinne des Kindeswohles setzt in vielen Fällen eine Ruhephase und die lebensweltliche Trennung der Parteien voraus. Zum Teil bedarf sie der Flucht in Frauenhäuser, Zufluchtswohnungen oder in private Netzwerke sowie der Geheimhaltung des Aufenthaltsortes. Eine Untersuchung des BMFSFJ $^{10}$ aus dem Jahr 2002 zum Sorge- und Umgangsrecht bei häuslicher Gewalt ergab, dass 70 Prozent der Frauen, deren Kinder Kontakt zum Vater hatten, während der Besuche oder der Übergabe erneut misshandelt wurden. 58 Prozent der Kinder erlitten Gewalt während der Umgangszeit mit dem nicht-sorgeberechtigten Elternteil. Weitere empirische Untersuchungen zeigen, dass gerade in der Trennungsphase das Gewalt- und Tötungsrisiko für Frauen und Kinder um ein Fünffaches höher ist. ${ }^{11}$ Das durch das FGG-RG geplante beschleunigte Verfahren mit dem am Konsens orientierten frü- hen ersten Termin und der zügigen Einleitung von Umgangskontakten findet genau in dieser schutzsensiblen Phase nach der Trennung statt.

\section{Gefahr der Verfestigung dysfunktionaler Strukturen}

Frauen und Kinder, die von Gewalt betroffen sind, zeigen häufig posttraumatische Belastungsstörungen. Deswegen besteht die Gefahr, dass sie vor Gericht - vor allem in einem so frühen Stadium nach der Trennung vom gewalttätigen Elternteil/Partner - nicht angemessen über die erlebte Gewalt berichten können (Panik/Kontrollverlust, Apathie, Dissoziieren, Bagatellisieren). Häufig besteht bei der Mutter auch die Angst, dass ihr Bedürfnis nach Schutz für sich und ihre Kinder und eine daraus resultierende Umgangsverweigerung im familiengerichtlichen Verfahren als mangelnde Erziehungskompetenz und fehlende Kooperationsbereitschaft negativ bewertet werden. Die im FGG-Reformgesetz vorgesehenen vier Wochen ab Antragseingang lassen weder für das Jugendamt noch für die auf die Unterstützung der Frauen und Kinder spezialisierten Beratungsstellen genügend Zeit, um die Betroffenen zu stabilisieren sowie die Lebenssituation und die Gewalterfahrung hinreichend zu explorieren. Ein nicht umfassend vorbereiteter früher erster Termin mit einer sich sofort anschließenden Einstweiligen Anordnung oder einer Einigung im Termin birgt daher die Gefahr, bestehende dysfunktionale Strukturen und Machtgefälle zu verfestigen.

Problematisch erscheint daher insgesamt ein undifferenziertes Beschleunigungsgebot, das sich lapidar an die Struktur des $\mathbb{6} 61 \mathrm{a}$ Abs. 2 ArbGG und an die Modellpraxis des Amtsgerichts Cochem anlehnt. Ziel sollte vielmehr sein, die mit dem FGG-RG nun vorgelegte Verfahrensordnung so zu entwickeln, dass dort, wo kindschaftsrechtliche Belange eröffnet sind, das Kindeswohl als vorrangiges Leit- und Strukturelement etabliert wird. In Verfahren, die sich mit Fragen des Umgangs- oder Sorgerechts oder auch der Kindeswohlgefährdung beschäftigen, sollte das Kind als tatsächliches Rechtssubjekt mit seinen - durch Grundgesetz und UN-Kinderrechtskonvention formulierten - Schutzbedürfnissen in den Blick rücken. Der Schutz von Frauen und Kindern vor Gewalt aber bedingt sich wechselseitig.

7 Pfeiffer, Christian, Wetzels, Peter: Kinder als Täter und Opfer. Eine Analyse auf der Basis der PKS und einer repräsentativen Opferbefragung, Hannover 1997.

8 Radford, Lorraine, Hester, Marianne: Mothering Through Domestic Violence, London 2006, S. 53 ff., Hester, Marianne et al.: Making an impact. Children and domestic violence. London 1998; Hester, Marianne, Pearson, Chris: From periphery to centre. Domestic violence in work with abused children, Bristol 1998.

9 Im Überblick: Kindler, Heinz: Partnergewalt und Beeinträchtigungen kindlicher Entwicklung. Ein Forschungsüberblick, in: Kavemann, Barbara, Kreyssig, Ulrike (Hg.): Handbuch Kinder und Häusliche Gewalt, Wiesbaden 2006, S. 36 ff.; Strasser, Philomena: Kinder legen Zeugnis ab. Gewalt gegen Frauen als Trauma für Kinder, Innsbruck 2001.

10 BMFSFJ: Sorge und Umgangsrecht bei häuslicher Gewalt: aktuelle rechtliche Empfehlungen, wissenschaftliche Erkenntnisse und Empfehlungen, Berlin 2002.

11 BMFSFJ: Lebenssituation, Sicherheit und Gesundheit von Frauen in Deutschland, Berlin 2004. 